\title{
ON ISOMORPHISMS OF CERTAIN FUNCTORS FOR CHEREDNIK ALGEBRAS
}

\author{
IVAN LOSEV
}

ABStRACT. Bezrukavnikov and Etingof introduced some functors between the categories $\mathcal{O}$ for rational Cherednik algebras. Namely, they defined two induction functors $\operatorname{Ind}_{b}$, ind $_{\lambda}$ and two restriction functors $\operatorname{Res}_{b}, \operatorname{res}_{\lambda}$. They conjectured that one has functor isomorphisms $\operatorname{Ind}_{b} \cong \operatorname{ind}_{\lambda}, \operatorname{Res}_{b} \cong \operatorname{res}_{\lambda}$. The goal of this paper is to prove this conjecture.

\section{INTRODUCTION}

The goal of this paper is to establish isomorphisms between certain functors arising in the representation theory of rational Cherednik algebras. These functors are parabolic induction and restriction functors introduced by Bezrukavnikov and Etingof in $\mathrm{BE}$.

Let us recall the definition of a rational Cherednik algebra that first appeared in EG]. The base field is the field $\mathbb{C}$ of complex numbers. Let $\mathfrak{h}$ be a finite dimensional vector space and $W \subset \mathrm{GL}(\mathfrak{h})$ a finite subgroup generated by the subset $S \subset W$ of complex reflections. By definition, a complex reflection is an element $s \in \mathrm{GL}(\mathfrak{h})$ of finite order with $\operatorname{rk}(s-\mathrm{id})=1$. For $s \in S$ pick elements $\alpha_{s}^{\vee} \in \operatorname{im}(s-\mathrm{id})$ and $\alpha_{s} \in(\mathfrak{h} / \operatorname{ker}(s-\mathrm{id}))^{*} \subset \mathfrak{h}^{*}$ with $\left\langle\alpha_{s}, \alpha_{s}^{\vee}\right\rangle=2$. Also pick a $W$-invariant map $c: S \rightarrow \mathbb{C}$. Define the rational Cherednik algebra $H\left(=H_{c}(W, \mathfrak{h})\right)$ as the quotient of $T\left(\mathfrak{h} \oplus \mathfrak{h}^{*}\right) \# W$ by the relations

$$
\begin{aligned}
& {\left[x, x^{\prime}\right]=0,} \\
& {\left[y, y^{\prime}\right]=0,} \\
& {[y, x]=\langle y, x\rangle-\sum_{s \in S} c(s)\left\langle\alpha_{s}, y\right\rangle\left\langle\alpha_{s}^{\vee}, x\right\rangle s,} \\
& x, x^{\prime} \in \mathfrak{h}^{*}, y, y^{\prime} \in \mathfrak{h} .
\end{aligned}
$$

We have the triangular decomposition $H=S(\mathfrak{h}) \otimes \mathbb{C} W \otimes \mathbb{C}[\mathfrak{h}]$. Using this decomposition one can introduce the category $\mathcal{O}:=\mathcal{O}_{c}(W, \mathfrak{h})$ for $H$ as the full subcategory of the category of left $H$-modules consisting of all modules $M$ satisfying the following two conditions:

(1) $M$ is finitely generated as a $\mathbb{C}[\mathfrak{h}]$-module.

(2) $\mathfrak{h}$ acts on $M$ locally nilpotently.

Now pick a parabolic subgroup $\underline{W} \subset W$, i.e., the stabilizer of some point in $\mathfrak{h}$. The space $\mathfrak{h}$ decomposes into the direct sum $\mathfrak{h}^{\underline{W}} \oplus \mathfrak{h}_{W}$, where $\mathfrak{h} \frac{W}{}$ stands for the space of $\underline{W}$-invariants in $\mathfrak{h}$, and $\mathfrak{h}_{\underline{W}}$ is a unique $\underline{W}$-stable complement to $\mathfrak{h} \underline{\underline{W}}$. Consider

Received by the editors January 25, 2012 and, in revised form, November 25, 2012.

2010 Mathematics Subject Classification. Primary 16G99.

The author was supported by the NSF grant DMS-0900907. 
the rational Cherednik algebra $\underline{H}^{+}:=H_{c}\left(\underline{W}, \mathfrak{h}_{W}\right)$, where, abusing the notation, $c$ stands for the restriction of $c$ to $S \cap \underline{W}$. Consider the category $\underline{\mathcal{O}}^{+}:=\mathcal{O}_{c}\left(\underline{W}, \mathfrak{h}_{\underline{W}}\right)$.

For $b \in \mathfrak{h}, \lambda \in \mathfrak{h}^{*}$ with $W_{b}=W_{\lambda}=\underline{W}$ Bezrukavnikov and Etingof in [BE], Subsection 3.5, defined the restriction functors $\operatorname{Res}_{b}, \operatorname{res}_{\lambda}: \mathcal{O} \rightarrow \underline{\mathcal{O}}^{+}$and the induction functors $\operatorname{Ind}_{b}$, ind $_{\lambda}: \underline{\mathcal{O}}^{+} \rightarrow \mathcal{O}$. The definitions will be recalled in Subsection 2.2 . The functors $\operatorname{Res}_{b}, \operatorname{Ind}_{b}$ do not depend on $b$ up to a (non-canonical) isomorphism, and a similar claim holds for $\operatorname{res}_{\lambda}$, ind $d_{\lambda}$. Conjecture 3.17 in $[\mathrm{BE}$ asserts that there are non-canonical isomorphisms $\operatorname{Res}_{b} \cong \operatorname{res}_{\lambda}, \operatorname{Ind}_{b} \cong \operatorname{ind}_{\lambda}$. In this paper we prove the conjecture. In particular, the conjecture implies that the functors $\operatorname{Res}_{b}$, $\operatorname{Ind}_{b}$ are bi-adjoint. This result was obtained earlier by Shan, [S], and used by her to equip the categories $\mathcal{O}$ with a categorification structure. One application of our result is that the induction and restriction functors (and hence the Shan categorification functors) preserve the subcategories of standardly filtered objects. This observation will be crucial to show that the corresponding categories $\mathcal{O}$ form a highest weight categorification; see [L2].

The paper is organized as follows.

In Section 2 we gather all necessary definitions and preliminary results. In Subsection 2.1 we recall the isomorphism of completions theorem of Bezrukavnikov and Etingof, $\mathrm{BE}$, Theorem 3.2. The functors of interest are defined using this isomorphism of completions. Their definitions are recalled in Subsection 3.1. In Subsection 2.3 we recall some other results on isomorphisms of completions obtained in [L1] that are used in the proof of the main result.

In Section 3 we prove an isomorphism of the functors $\operatorname{Res}_{b}$, res $_{\lambda}$. In Subsection 3.1 we introduce some auxiliary functors $\operatorname{Res}_{b, \lambda}, \operatorname{res}_{b, \lambda}$ such that $\operatorname{Res}_{b, \lambda} \cong \operatorname{Res}_{b}, \operatorname{res}_{0, \lambda} \cong$ $\operatorname{res}_{\lambda}$. Our strategy is to establish embeddings $\operatorname{res}_{b, \lambda} \hookrightarrow \operatorname{Res}_{b, 0}, \operatorname{res}_{0, \lambda} \hookrightarrow \operatorname{res}_{b, \lambda}$. We can establish the latter directly, this is done in Subsection 3.4. However, we arrive at some convergence issue with the former embedding. To fix these issues we need to work with algebras and modules not over $\mathbb{C}$ but over $\left.R:=\mathbb{C}\left[t^{-1}, t\right]\right]$. We treat this case in Subsection 3.2 and then establish an embedding $\operatorname{res}_{b, \lambda} \hookrightarrow \operatorname{Res}_{b, 0}$ in Subsection 3.3. Finally, in Subsection 3.5 we show that the resulted embedding res $_{0, \lambda} \hookrightarrow \operatorname{Res}_{b, 0}$ is actually an isomorphism.

The proof of an isomorphism $\operatorname{Ind}_{b} \cong \operatorname{ind}_{\lambda}$ is similar to that of $\operatorname{Res}_{b} \cong \operatorname{res}_{\lambda}$. In Section 4 we explain necessary modifications.

\section{Preliminaries}

2.1. Isomorphisms of completions, I. In this subsection we will recall some results from $\mathrm{BE}$ ] related to isomorphisms of completions of different rational Cherednik algebras. Namely, we define the completions $H^{\wedge_{b}}, H^{\wedge_{\lambda}}, \underline{H}^{\wedge_{b}}, \underline{H}^{\wedge_{\lambda}}$, where $\underline{H}:=$ $H_{c}(\underline{W}, \mathfrak{h})$ and describe isomorphisms between $H^{\wedge_{b}}$ (resp., $H^{\wedge_{\lambda}}$ ) and some matrix algebra with coefficients in $\underline{H}^{\wedge_{b}}$ (resp, in $\underline{H}^{\wedge_{\lambda}}$ ).

Pick a point $b \in \mathfrak{h}$ with $W_{b}=\underline{W}$. Let $\mathbb{C}[\mathfrak{h}]^{\wedge_{W b}}$ denote the completion of $\mathbb{C}[\mathfrak{h}]$ at $W b$. Define the completion $H^{\wedge_{b}}:=\mathbb{C}[\mathfrak{h}]^{\wedge_{W b}} \otimes_{\mathbb{C}[\mathfrak{h}]} H$ of $H$ at $b$. The space $H^{\wedge_{b}}$ comes equipped with a topology, and has a unique topological algebra structure extended from $H$ by continuity.

Similarly, we can define the completion $\underline{H}^{\wedge_{b}}:=\mathbb{C}[\mathfrak{h}]^{\wedge_{b}} \otimes_{\mathbb{C}[\mathfrak{h}]} \underline{H}$ of $\underline{H}$ at $b$.

A relation between $H^{\wedge_{b}}$ and $\underline{H}^{\wedge_{b}}$ is as follows. In BE], Subsection 3.2, for finite groups $G_{0} \subset G$ and an algebra $A$ containing $\mathbb{C} G_{0}$, Bezrukavnikov and Etingof considered the right $A$-module $\operatorname{Fun}_{G_{0}}(G, A)$ of $G_{0}$-equivariant maps $G \rightarrow A$. Then 
they defined the centralizer algebra $Z\left(G, G_{0}, A\right)$ as the endomorphism algebra of the right $A$-module $\operatorname{Fun}_{G_{0}}(G, A)$. Below we write $\mathrm{C}(\bullet)$ for $Z(W, \underline{W}, \bullet)$.

The following proposition is a slightly modified version of $[\mathrm{BE}$, Theorem 3.2.

Proposition 2.1. There is a unique continuous homomorphism $\vartheta_{b}: H^{\wedge_{b}} \rightarrow$ $\mathrm{C}\left(\underline{H}^{\wedge_{b}}\right)$ such that

$$
\begin{aligned}
& {\left[\vartheta_{b}(u) f\right](w)=f(w u),} \\
& {\left[\vartheta_{b}\left(x_{\alpha}\right) f\right](w)=\underline{x}_{w \alpha} f(w),} \\
& {\left[\vartheta_{b}\left(y_{a}\right) f\right](w)=\underline{y}_{w a} f(w)+\sum_{s \in S \backslash W_{b}} \frac{2 c(s)}{1-\lambda_{s}} \frac{\alpha_{s}(w a)}{\underline{x}_{\alpha_{s}}}(f(s w)-f(w)),} \\
& u, w \in W, \alpha \in \mathfrak{h}^{*}, a \in \mathfrak{h}, f \in \operatorname{Fun}_{\underline{W}}\left(W, \underline{H}^{\wedge_{b}}\right) .
\end{aligned}
$$

This homomorphism is an isomorphism of topological algebras.

Here $x_{\alpha}, \underline{x}_{\alpha}$ denote the elements of $H, \underline{H}$ corresponding to $\alpha \in \mathfrak{h}^{*}, y_{a}, \underline{y}_{a}$ have a similar meaning. Of course, when one views $\frac{1}{\underline{x}_{\alpha_{s}}}$ as an element of $\mathbb{C}[\mathfrak{h}]^{\wedge_{b}}$, one expands this fraction near $b$. By $\lambda_{s}$ we denote a unique non-unit eigenvalue for the action of $s$ on $\mathfrak{h}^{*}$.

The completion $\underline{H}^{\wedge_{b}}$ is naturally isomorphic to the completion $\underline{H}^{\wedge_{0}}:=\mathbb{C}[\mathfrak{h}]^{\wedge_{0}}$ $\otimes_{\mathbb{C}[\mathfrak{h}]} \underline{H}$. An isomorphism $\underline{H}^{\wedge_{b}} \stackrel{\sim}{\rightarrow} \underline{H}^{\wedge_{0}}$ is given by $w \mapsto w, \underline{x}_{\alpha} \mapsto \underline{x}_{\alpha}-\langle b, \alpha\rangle$, $\underline{y}_{a} \mapsto \underline{y}_{a}$.

Similarly, one can consider the completions $H^{\wedge_{\lambda}}, \underline{H}^{\wedge_{\lambda}}$ at $\lambda \in \mathfrak{h}^{*}$ with $W_{\lambda}=\underline{W}$. Then one has an isomorphism $\widetilde{\vartheta}_{\lambda}: H^{\wedge_{\lambda}} \rightarrow \mathrm{C}\left(\underline{H}^{\wedge_{\lambda}}\right)$. It is given by

$$
\begin{aligned}
& {\left[\widetilde{\vartheta}_{\lambda}(u) f\right](w)=f(w u),} \\
& {\left[\widetilde{\vartheta}_{\lambda}\left(x_{\alpha}\right) f\right](w)=\underline{x}_{w \alpha} f(w)-\sum_{s \in S \backslash W_{\lambda}} \frac{2 c_{s}}{1-\lambda_{s}^{-1}} \frac{\alpha_{s}^{\vee}(w a)}{\underline{y}_{\alpha_{s}^{\vee}}}(f(s w)-f(w)),} \\
& {\left[\widetilde{\vartheta}_{\lambda}\left(y_{a}\right) f\right](w)=\underline{y}_{w a} f(w) .}
\end{aligned}
$$

We remark that both completions we considered were "partial": we allowed power series either only in $x$ 's or only in $y$ 's. If we allow both, then the product will not be well defined.

2.2. Definition of functors. In this subsection we will introduce exact functors $\operatorname{Res}_{b}, \operatorname{res}_{\lambda}: \mathcal{O} \rightarrow \underline{\mathcal{O}}^{+}, \operatorname{Ind}_{b}, \operatorname{ind}_{\lambda}: \underline{\mathcal{O}}^{+} \rightarrow \mathcal{O}$ for $b \in \mathfrak{h}, \lambda \in \mathfrak{h}^{*}$ with $W_{b}=W_{\lambda}=\underline{W}$.

We need to define some auxiliary categories of $H, \underline{H}, \underline{H}^{+}$-modules. For $\mu \in \mathfrak{h}^{*}$ consider the category $\mathcal{O}^{\mu}$ consisting of all $H$-modules $M$ satisfying

(1) $M$ is finitely generated over $S\left(\mathfrak{h}^{*}\right)$.

(2) $S(\mathfrak{h})^{W}$ acts on $M$ with generalized eigencharacter $\mu$.

It is easy to see that $\mathcal{O}^{0}=\mathcal{O}$. More generally, one can consider the category $\widetilde{\mathcal{O}}^{\mu}$ of all $H$-modules satisfying (2). Any module in $\widetilde{\mathcal{O}}^{\mu}$ is the direct limit of modules in $\mathcal{O}^{\mu}$. Similarly, we have the categories $\underline{\widetilde{\mathcal{O}}}, \underline{\widetilde{\mathcal{O}}}^{+}$.

Now let us recall the definitions of the functors $\operatorname{Res}_{b}, \operatorname{res}_{\lambda}, \operatorname{Ind}_{b}$, ind $_{\lambda}$ from $[\mathrm{BE}$.

First, we define $\operatorname{Res}_{b}$. Pick $M \in \mathcal{O}$ and consider its completion $M^{\wedge_{b}}:=$ $\mathbb{C}[\mathfrak{h}]^{\wedge_{W b}} \otimes_{\mathbb{C}[\mathfrak{h}]} M$ at $W b$. Then $M^{\wedge_{b}}$ is an $H^{\wedge_{b}}$-module and hence we can consider the push-forward $\vartheta_{b *}\left(M^{\wedge_{b}}\right)$ that is a $\mathrm{C}\left(\underline{H}^{\wedge_{b}}\right)$-module. There is a natural equivalence $I: \underline{H}^{\wedge_{b}}$-Mod $\stackrel{\sim}{\rightarrow} \mathrm{C}\left(\underline{H}^{\wedge_{b}}\right)$-Mod; see $[\mathrm{BE}$, Subsection 3.2. So we get 
an $\underline{H}^{\wedge_{b}}$-module $I^{-1} \circ \vartheta_{b *}\left(M^{\wedge_{b}}\right)$. For an $\underline{H}^{\wedge_{b}}$-module $N^{\prime}$ and $\lambda \in \mathfrak{h}^{*} \underline{W}$ let $\underline{E}_{\lambda}\left(N^{\prime}\right)$ stand for the space of vectors annihilated by $\left(\underline{y}_{a}-\langle\lambda, a\rangle\right)^{n}$ for all $a \in \mathfrak{h}$ and $n \gg 0$. For an $\underline{H}$-module $N$ set

$$
\zeta_{\lambda}(N):=\bigcap_{a \in \mathfrak{h}^{*} \underline{W}} \operatorname{ker}\left(\underline{y}_{a}-\langle\lambda, a\rangle\right) .
$$

We set $\operatorname{Res}_{b}(M):=\zeta_{0} \circ \underline{E}_{0} \circ I^{-1} \circ \vartheta_{b *}\left(M^{\wedge_{b}}\right)$.

The proof of the following lemma is easy (compare with Lemma 3.4 below).

Lemma 2.2. For any $\lambda \in \mathfrak{h}^{* \underline{W}}$ the functor $\zeta_{\lambda} \circ \underline{E}_{\lambda}$ is an equivalence

- from the category of $\underline{H}^{\wedge_{b}}$-modules that are finitely generated over $\mathbb{C}[\mathfrak{h}]^{\wedge_{b}}$,

- to the category $\underline{\mathcal{O}}^{+}$.

Moreover, the functors $\zeta_{\lambda} \circ \underline{E}_{\lambda}$ are naturally isomorphic for all $\lambda \in \mathfrak{h}^{*} \underline{W}$.

Let us construct a functor $\operatorname{Ind}_{b}: \underline{\mathcal{O}}^{+} \rightarrow \mathcal{O}$. We have an equivalence $\mathcal{F}:=$ $\vartheta_{b *}^{-1} \circ I \circ \underline{E}_{0}^{-1} \circ \zeta_{0}^{-1}$ from $\underline{\mathcal{O}}^{+}$to the category $\mathcal{O}^{\wedge_{b}}$ of $H^{\wedge_{b}}$-modules that are finitely generated over $\mathbb{C}[\mathfrak{h}]^{\wedge}{ }^{W b}$. Now for an $H$-module $M^{\prime}$ let $E_{\lambda}\left(M^{\prime}\right)$ be the generalized eigenspace of $S(\mathfrak{h})^{W}$ with eigenvalue $\lambda: S(\mathfrak{h})^{W} \rightarrow \mathbb{C}$. Set $\operatorname{Ind}_{b}(N):=E_{0} \circ \mathcal{F}(N)$. In $\left[\mathrm{BE}\right.$, Subsection 3.5, it was shown that $\operatorname{Ind}_{b}\left(\underline{\mathcal{O}}^{+}\right) \subset \mathcal{O}$ (a priori, one only sees that $\left.\operatorname{Ind}_{b}\left(\underline{\mathcal{O}}^{+}\right) \subset \widetilde{\mathcal{O}}\right)$ and that $\operatorname{Ind}_{b}$ is exact and right adjoint to $\operatorname{Res}_{b}$.

Proceed to the definition of $\operatorname{res}_{\lambda}$. Pick $M \in \mathcal{O}$. Consider the completion $M^{\wedge_{0}}$. Consider the $H$-module $E_{\lambda}\left(M^{\wedge_{0}}\right)$. The $H$-action on this module extends to $H^{\wedge_{\lambda}}$. So we can consider the push-forward $\widetilde{\vartheta}_{\lambda *} \circ E_{\lambda}\left(M^{\wedge_{0}}\right)$ and also the $\underline{H}^{\wedge^{\lambda}}$-module $N^{\prime}:=I^{-1} \circ \widetilde{\vartheta}_{\lambda *} \circ E_{\lambda}\left(M^{\wedge_{0}}\right)$. The operators $\underline{y}_{a}$ act with generalized eigenvalue $\lambda$ on $N^{\prime}$, in other words, $N^{\prime} \in \widetilde{\widetilde{\mathcal{O}}}^{\lambda}$.

The proof of the following lemma is again easy.

Lemma 2.3. The functor $\zeta_{\lambda}$ is an isomorphism $\underline{\widetilde{\mathcal{O}}}^{\lambda} \rightarrow \underline{\widetilde{\mathcal{O}}}^{+}$.

So we set $\operatorname{res}_{\lambda}(M):=\zeta_{\lambda} \circ I^{-1} \circ \tilde{\vartheta}_{\lambda *} \circ E_{\lambda}\left(M^{\wedge_{0}}\right)$.

To define ind $\lambda_{\lambda}$ we reverse the procedure. Pick $N \in \underline{\mathcal{O}}^{+}$. Then, according to [BE], Corollary 3.3, $M_{0}:=\widetilde{\vartheta}_{\lambda *}^{-1} \circ I \circ \zeta_{\lambda}^{-1}(N) \in \mathcal{O}^{\lambda}$. Set $\operatorname{ind}_{\lambda}(N):=E_{0}\left(M_{0}^{\wedge_{0}}\right)$.

The functors $\operatorname{res}_{\lambda}$, ind $_{\lambda}$ were constructed in $\mathrm{BE}$. In fact, their initial definition was quite different, but $\mathrm{BE}$, Proposition 3.13, established an equivalence with the definition given above. From the initial definition of $[\mathrm{BE}]$ it follows that $\operatorname{res}_{\lambda}$, $\operatorname{ind}_{\lambda}$ are exact, their images lie in $\underline{\mathcal{O}}^{+}, \mathcal{O}$, respectively, and ind ${ }_{\lambda}$ is left adjoint to res ${ }_{\lambda}$.

2.3. Isomorphisms of completions, II. In this section we will explain some results from [L1]. In [L1] we worked with the homogenized versions of the algebras. More precisely, define the $\mathbb{C}[h]$-algebra $H_{h}$ as the quotient of $T\left(\mathfrak{h} \oplus \mathfrak{h}^{*}\right)[h] \# W$ by the homogeneous versions of the relations (1), namely with the third relation replaced with

$$
[y, x]=h\left(\langle y, x\rangle-\sum_{s \in S} c(s)\left\langle\alpha_{s}, y\right\rangle\left\langle\alpha_{s}^{\vee}, x\right\rangle s\right) .
$$

We can sheafify $H_{h}$ over $\mathfrak{h} \oplus \mathfrak{h}^{*} / W$ (compare with [L1], Subsection 2.4) using a procedure similar to the formal microlocalization. We get a pro-coherent sheaf $\mathcal{H}_{h}$ of $\mathbb{C}[[h]]$-algebras on $\mathfrak{h} \oplus \mathfrak{h}^{*} / W$.

Similarly, picking a parabolic subgroup $\underline{W} \subset W$ one can define a $\mathbb{C}[h]$-algebra $\underline{H}_{h}$ and sheafify it over $\mathfrak{h} \oplus \mathfrak{h}^{*} / \underline{W}$ to get a sheaf $\underline{\mathcal{H}}_{h}$. 
Let $\pi: \mathfrak{h} \oplus \mathfrak{h}^{*} \rightarrow \mathfrak{h} \oplus \mathfrak{h}^{*} / W$ denote the quotient morphism. Consider the locally closed subvariety of $\mathfrak{h} \oplus \mathfrak{h}^{*}$ consisting of all points $(b, \lambda)$ with $W_{(b, \lambda)}=W$. Let $\mathcal{L}$ denote the image of this subvariety in $\mathfrak{h} \oplus \mathfrak{h}^{*} / W$. Then $\mathcal{L}$ is a symplectic leaf of the Poisson variety $\mathfrak{h} \oplus \mathfrak{h}^{*} / W$.

As in [L1], Subsection 2.4, we can define the completion $\mathcal{H}_{h}^{\wedge \mathcal{L}}$ of the sheaf $\mathcal{H}_{h}$ along $\mathcal{L}$ and its sheaf-theoretic restriction $\left.\mathcal{H}_{h}^{\wedge \mathcal{L}}\right|_{\mathcal{L}}$ to $\mathcal{L}$.

Similarly, we can define the open subvariety $\underline{\mathcal{L}} \subset \mathfrak{h} \underline{\underline{W}} \oplus \mathfrak{h}^{*} \underline{W} \subset\left(\mathfrak{h} \oplus \mathfrak{h}^{*}\right) / \underline{W}$ and the completion $\left.\underline{\mathcal{H}}_{h}^{\wedge}\right|_{\mathcal{L}}$. We remark that $\mathcal{L}$ is naturally identified with the quotient $\underline{\mathcal{L}} / \Xi$, where $\Xi:=N_{W}(\underline{W}) / \underline{W}$.

The sheaves we have introduced come equipped with certain group actions. First, let us note that the 2-dimensional torus $\left(\mathbb{C}^{\times}\right)^{2}$ acts on $H_{h}:\left(z_{1}, z_{2}\right) \cdot w=$ $w,\left(z_{1}, z_{2}\right) \cdot x=z_{1} x,\left(z_{1}, z_{2}\right) \cdot y=z_{2} y,\left(z_{1}, z_{2}\right) \cdot h=z_{1} z_{2} h, w \in W \subset H_{h}, x \in \mathfrak{h}^{*} \subset$ $H_{h}, y \in \mathfrak{h} \subset H_{h}$. This $\left(\mathbb{C}^{\times}\right)^{2}$-action extends to actions on $\mathcal{H}_{h}, \mathcal{H}_{h}^{\wedge \mathcal{L}},\left.\mathcal{H}_{h}^{\wedge \mathcal{L}}\right|_{\mathcal{L}}$ by sheaf of algebras automorphisms.

The sheaf $\left.\underline{\mathcal{H}}_{h}^{\wedge}\right|_{\underline{\mathcal{L}}}$ also carries a similar $\left(\mathbb{C}^{\times}\right)^{2}$-action. Moreover, $\underline{H}_{h}$ is acted on by $N_{W}(\underline{W})$ (the action is being induced from the natural $N_{W}(\underline{W})$-action on $\mathfrak{h} \oplus \mathfrak{h}^{*}$ ). This action again extends to $\left.\underline{\mathcal{H}}_{h}^{\wedge}\right|_{\mathcal{L}}$.

Consider the sheaf $\mathrm{C}\left(\left.\underline{\mathcal{H}}_{h}^{\wedge}\right|_{\underline{\mathcal{L}}}\right)$ on $\underline{\mathcal{L}}$. There is a natural action of $\Xi$ on this sheaf by algebra automorphisms; see [L1], Subsection 2.3. Let $\rho: \underline{\mathcal{L}} \rightarrow \mathcal{L}$ denote the projection (i.e., the quotient by $\Xi)$. Abusing the notation we write $\mathrm{C}\left(\left.\underline{\mathcal{H}}_{h}^{\wedge}\right|_{\underline{\mathcal{L}}}\right)^{\Xi}$ instead of $\rho_{*}\left(\mathrm{C}\left(\underline{\mathcal{H}}_{h}^{\wedge} \underline{\mathcal{L}}_{\underline{\mathcal{L}}}\right)\right)^{\Xi}$. This is a sheaf of algebras on $\mathcal{L}$.

So we have constructed two sheaves of algebras $\left.\mathcal{H}_{h}^{\wedge \mathcal{L}}\right|_{\mathcal{L}}, \mathrm{C}\left(\left.\underline{\mathcal{H}}_{h}^{\wedge}\right|_{\mathcal{L}}\right){ }^{\Xi}$ on $\mathcal{L}$. These sheaves are not isomorphic but they become isomorphic if we twist one of them by a 1-cocycle of inner automorphisms. More precisely, let us fix an open covering $\bigcup_{i} U_{i}$ of $\mathcal{L}$ by $\left(\mathbb{C}^{\times}\right)^{2}$-stable open affine subvarieties.

Proposition 2.4. There are $\left(\mathbb{C}^{\times}\right)^{2}$-equivariant $\mathbb{C}[[h]]$-linear isomorphisms

$$
\Theta^{i}:\left.\mathcal{H}_{h}^{\wedge \mathcal{L}}\right|_{\mathcal{L}}\left(U_{i}\right) \rightarrow \mathrm{C}\left(\left.\underline{\mathcal{H}}_{h}^{\wedge} \underline{\underline{\mathcal{L}}}\right|_{\underline{\mathcal{L}}}\right)^{\Xi}\left(U_{i}\right)
$$

and $\left(\mathbb{C}^{\times}\right)^{2}$-invariant elements

$$
X_{i j} \in \mathfrak{z}^{h}\left(\mathrm{C}\left(\underline{\mathcal{H}}_{h}^{\wedge} \underline{\mathcal{L}}_{\underline{\mathcal{L}}}\right)^{\Xi}\right)\left(U_{i j}\right),
$$

where $U_{i j}:=U_{i} \cap U_{j}$, such that:

(1) Modulo $h$ the isomorphism $\Theta^{i}$ coincides with the natural isomorphism $\Theta_{0}$ : $\left.\left(S\left(\mathfrak{h} \oplus \mathfrak{h}^{*}\right) \# W\right)^{\wedge} \mathcal{L}\right|_{\mathcal{L}} \rightarrow\left(\left.\mathrm{C}\left(S\left(\mathfrak{h} \oplus \mathfrak{h}^{*}\right) \# \underline{W}\right)^{\wedge} \underline{\mathcal{L}}\right|_{\mathcal{L}}\right)^{\Xi}$; see [L1], Subsection 2.5.

(2) $\Theta^{i}=\exp \left(\operatorname{ad} X_{i j}\right) \Theta^{j}$ for all $i, j$.

Here for a $\mathbb{C}[h]$-algebra $A$ by $\mathfrak{z}^{h}(A)$ we denote the preimage of the center of $A / h A$ in $A$.

This proposition is a weaker version of Theorem 2.5.3 in [L1] (in fact, in that theorem we have only $\mathbb{C}^{\times}$-actions, but the proof extends directly to the sheaves with $\left(\mathbb{C}^{\times}\right)^{2}$-actions).

We will apply Proposition 2.4 in the following situation. Let $U_{1}:=\left[\left(\mathfrak{h} \frac{W}{}\right)^{r} \times\right.$ $\left.\mathfrak{h}^{*} \underline{W}\right] / \Xi, U_{2}:=\left[\mathfrak{h} \underline{\underline{W}} \times\left(\mathfrak{h}^{*} \underline{W}\right)^{r}\right] / \Xi \subset \mathcal{L}$, where $(\mathfrak{h} \underline{W})^{r},(\mathfrak{h} * \underline{W})^{r}$ denote the open subsets of all points with stabilizer exactly $\underline{W}$. Consider the algebra $\left.\mathcal{H}_{h}^{\wedge \mathcal{L}}\right|_{\mathcal{L}}\left(U_{1}\right)$. We can complete $H_{h}$ at $b$ : take the ideal $\mathfrak{m}_{b} \subset H_{h}$, compare with [L1, Subsection 1.2, and set $H_{h}^{\wedge_{b}}:=\lim _{n \rightarrow \infty} H_{h} / \mathfrak{m}_{b}^{n}$. In fact, the natural homomorphism $H_{h} \rightarrow H_{h}^{\wedge_{b}}$ factors through $\mathcal{H}_{h}^{\wedge} \overleftarrow{\mathcal{L}}_{\mathcal{L}}\left(U_{1}\right)$. Moreover, $H_{h}^{\wedge}$ is the completion of $\left.\mathcal{H}_{h}^{\wedge \mathcal{L}}\right|_{\mathcal{L}}\left(U_{1}\right)$ with respect to the ideal analogous to $\mathfrak{m}_{b} \subset H_{h}$; compare with [L1], Subsection 2.5. 
A similar construction works for $\underline{H}_{h}$ so we get the completion $\underline{H}_{h}^{\wedge}$. We conclude that $\Theta^{1}$ induces an isomorphism $\Theta_{b}: H_{h}^{\wedge_{b}} \stackrel{\sim}{\rightarrow} \mathrm{C}\left(\underline{H}_{h}^{\wedge_{b}}\right)$. We remark that this isomorphism is equivariant with respect to the second copy of $\mathbb{C}^{\times}$in $\left(\mathbb{C}^{\times}\right)^{2}$ (the one acting on the $y$ 's).

Note, however, that we can produce an isomorphism $H_{h}^{\wedge_{b}} \rightarrow \mathrm{C}\left(\underline{H}_{h}^{\wedge_{b}}\right)$ by taking a homogeneous version of $\vartheta_{b}$. Namely, define $\vartheta_{b}$ on the generators of $H_{h}$ by

$$
\begin{aligned}
& {\left[\vartheta_{b}(u) f\right](w)=f(w u),} \\
& {\left[\vartheta_{b}\left(x_{\alpha}\right) f\right](w)=\underline{x}_{w \alpha} f(w),} \\
& {\left[\vartheta_{b}\left(y_{a}\right) f\right](w)=\underline{y}_{w a} f(w)+h \sum_{s \in S \backslash W_{b}} \frac{2 c_{s}}{1-\lambda_{s}} \frac{\alpha_{s}(w a)}{\underline{x}_{\alpha_{s}}}(f(s w)-f(w)) .}
\end{aligned}
$$

Then $\vartheta_{b}$ uniquely extends to a topological algebra isomorphism $H_{h}^{\wedge_{b}} \rightarrow \mathrm{C}\left(\underline{H}_{h}^{\wedge_{b}}\right)$. We remark that $\vartheta_{b}$ is also $\mathbb{C}^{\times}$-equivariant.

Lemma 2.5. There is an invertible element $X \in \mathbb{C}[\mathfrak{h} / \underline{W}]^{\wedge_{b}}$ such that $\Theta_{b}=\operatorname{Ad}(X) \circ$ $\vartheta_{b}$.

Proof. This follows from [L1], Lemma 5.2.1.

Being $\mathbb{C}^{\times}$-equivariant both $\Theta_{b}, \vartheta_{b}$ restrict to isomorphisms between the subalgebras in $H_{h}^{\wedge_{b}}, \mathrm{C}\left(\underline{H}_{h}^{\wedge_{0}}\right)$ consisting of all $\mathbb{C}^{\times}$-finite vectors ( "Cำ a finite dimensional $\mathbb{C}^{\times}$-stable subspace"). Take the quotient of these subalgebras by $h-1$. We get the algebras $H^{\wedge_{b}}, \mathrm{C}\left(\underline{H}^{\wedge_{b}}\right)$. Let $\theta_{b}$ denote the isomorphism of these algebras induced by $\Theta_{b}$. We still have the equality $\theta_{b}=\operatorname{Ad}(X) \circ \vartheta_{b}$.

Applying the same considerations to $U_{2}$, we get the isomorphism

$$
\widetilde{\theta}_{\lambda}: H^{\wedge_{\lambda}} \rightarrow \mathrm{C}\left(\underline{H}^{\wedge_{\lambda}}\right)
$$

and the invertible element $\tilde{X} \in \mathbb{C}\left[\mathfrak{h}^{*} / \underline{W}\right]^{\wedge_{\lambda}}$ with $\widetilde{\theta}_{\lambda}=\operatorname{Ad}(\widetilde{X}) \circ \widetilde{\vartheta}_{\lambda}$.

\section{ISOMORPHISM OF THE RESTRICTION FUNCTORS}

3.1. Functors $\operatorname{Res}_{b, \lambda}, \operatorname{res}_{b, \lambda}$. Let $b \in \mathfrak{h} \frac{W}{}, \lambda \in \mathfrak{h}^{*} \underline{W}$.

Suppose $W_{b}=\underline{W}$. Let us define a functor $\operatorname{Res}_{b, \lambda}: \mathcal{O} \rightarrow \underline{\mathcal{O}}^{+}$by

$$
\operatorname{Res}_{b, \lambda}(M)=\zeta_{\lambda} \circ I^{-1} \circ \underline{E}_{\lambda} \circ\left(\theta_{b}\right)_{*}\left(M^{\wedge_{b}}\right) .
$$

Here the functor $\underline{E}_{\lambda}$ on the category of $\mathrm{C}(\underline{H})$-modules is defined as before using the natural embedding $\mathbb{C}\left[\mathfrak{h}^{*}\right] \underline{W} \hookrightarrow \underline{H} \underline{\underline{W}} \hookrightarrow \mathrm{C}(\underline{H})$ (see [L1], Subsection 2.3).

Lemma 3.1. There is an isomorphism $\operatorname{Res}_{b, \lambda} \cong \operatorname{Res}_{b}$ for all $\lambda$.

Proof. First, let us remark that $\underline{E}_{\lambda}$ and $I^{-1}$ commute. Since $\zeta_{\lambda} \circ \underline{E}_{\lambda}$ is isomorphic to $\zeta_{0} \circ \underline{E}_{0}$ (Lemma 2.2), we see that $\operatorname{Res}_{b, \lambda} \cong \operatorname{Res}_{b, 0}$.

Recall $X$ from Lemma 2.5. The existence of $X$ implies that the functors $\left(\theta_{b}\right)_{*}$ and $\left(\vartheta_{b}\right)_{*}$ between the categories of $H^{\wedge_{b}}$ and of $\mathrm{C}\left(\underline{H}^{\wedge_{b}}\right)$-modules are isomorphic. So $\operatorname{Res}_{b, 0} \cong \operatorname{Res}_{b}$.

In fact, it will be useful for us to rewrite the definition of $\operatorname{Res}_{b, \lambda}$ a little bit. Namely, for an $H^{\wedge^{b}}$-module $M$ let $\underline{E}_{\lambda}^{\theta}$ denote the generalized eigenspace of the algebra $\mathbb{C}\left[\mathfrak{h}^{*} / \underline{W]}\right.$ with eigenvalue $\lambda$, where $\mathbb{C}\left[\mathfrak{h}^{*} / \underline{W]}\right.$ acts on $M$ via $\theta_{b}^{-1}$. So we have

$$
\operatorname{Res}_{b, \lambda}(M)=\zeta_{\lambda} \circ I^{-1} \circ\left(\theta_{b}\right)_{*} \circ \underline{E}_{\lambda}^{\theta}\left(M^{\wedge_{b}}\right) .
$$

The definition of $\operatorname{res}_{b, \lambda}$ is more technical. Let $W_{\lambda}=\underline{W}$. 
Below we will need certain "Euler elements" in $H, \underline{H}, \underline{H}^{+}$; see GGOR, Subsection 3.1. Pick some basis $y_{1}, \ldots, y_{n} \in \mathfrak{h} \subset H$ and let $x_{1}, \ldots, x_{n} \in \mathfrak{h}^{*}$ be the dual basis. We set

$$
\mathrm{eu}:=\sum_{i=1}^{n} x_{i} y_{i}+\frac{\operatorname{dim} \mathfrak{h}}{2}-\sum_{s \in S} \frac{2 c(s)}{1-\lambda_{s}} s .
$$

This element does not depend on the choice of $y_{1}, \ldots, y_{n}$, is $W$-invariant and satisfies the commutation relations $[\mathrm{eu}, x]=x,[\mathrm{eu}, y]=-y, x \in \mathfrak{h}^{*}, y \in \mathfrak{h}$.

Similarly, we can introduce the Euler elements $\underline{\mathrm{eu}} \in \underline{H}, \underline{\mathrm{eu}}^{+} \in \underline{H}^{+}$.

For a topological $H$-module $M$ consider the subspace $M^{\odot} \subset M$, whose elements, by definition, are sums $\sum_{a \in \mathbb{C}} \sum_{i \geqslant 0} m_{a, i}$, where

- the first sum is finite,

- there is $N_{a}$ such that $(\mathrm{eu}-a-i)^{N_{a}} m_{a, i}=0$ for all $i$, and

- the sum $\sum_{i \geqslant 0} m_{a, i}$ converges for every $a$.

Then $M^{\odot}$ is an $H$-submodule in $M$. For example, let $M \in \mathcal{O}$. Consider the completion $M^{\wedge_{0}}$ at 0 . The element eu acts diagonalizably on any simple module in $\mathcal{O}$. Since any object in $\mathcal{O}$ has finite length it follows that eu acts locally finitely on $M$. From here it is easy to see that $M^{\wedge_{0} \odot}=M^{\wedge_{0}}$.

For $M \in \mathcal{O}$ we set

$$
\operatorname{res}_{b, \lambda}(M)=\zeta_{\lambda} \circ I^{-1} \circ\left(\widetilde{\theta}_{\lambda}\right)_{*} \circ E_{\lambda}\left(M^{\wedge_{b} \odot}\right) .
$$

By construction, the operators $\underline{y}_{a}$ act on $I^{-1} \circ\left(\widetilde{\theta}_{\lambda}\right)_{*} \circ E_{\lambda}\left(M^{\wedge_{b}}\right)$ with generalized eigenvalue $\lambda$, so $\operatorname{res}_{b, \lambda}(M) \in \underline{\mathcal{\mathcal { O }}}^{+}$. Later we will see that $\operatorname{res}_{b, \lambda}(M)$ is actually in $\underline{\mathcal{O}}^{+}$.

Lemma 3.2. We have $\operatorname{res}_{0, \lambda}=\operatorname{res}_{\lambda}$.

Proof. This follows from the equality $M^{\wedge_{0}} \odot=M^{\wedge_{0}}$ and the existence of an element $\widetilde{X} \in \mathbb{C}[\mathfrak{h} / \underline{W}]^{\wedge_{\lambda}}$, compare with the proof of Lemma 3.1 .

We remark that $E_{\lambda}(M)$ coincides with the generalized eigenspace of $S(\mathfrak{h}) \frac{W}{}$ with

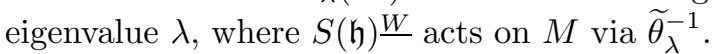

Below we will show that $\operatorname{res}_{0, \lambda} \hookrightarrow \operatorname{res}_{b, \lambda}$ and $\operatorname{res}_{b, \lambda} \hookrightarrow \operatorname{Res}_{b}$. The first embedding is established in Subsection 3.4. The proof is not very complicated, although it is somewhat unsatisfactory because it works only for the field $\mathbb{C}$ (perhaps it should be possible to make the same ideas work over an arbitrary algebraically closed field of characteristic 0 , but we do not know how). The embedding $\operatorname{res}_{b, \lambda} \hookrightarrow \operatorname{Res}_{b, \lambda}$ is more complicated. Let us explain where the complications come from.

Basically, we need to produce an embedding

$$
\left(\widetilde{\theta}_{\lambda}\right)_{*} \circ E_{\lambda} \circ\left(\bullet^{\wedge_{b} \odot}\right) \hookrightarrow\left(\theta_{b}\right)_{*} \circ \underline{E}_{\lambda}^{\theta}\left(\bullet^{\wedge_{b}}\right)
$$

of functors $\mathcal{O} \rightarrow \underline{\mathcal{O}}^{\lambda}$. That is, for $M \in \mathcal{O}$ we need to construct a functorial embedding $\Upsilon_{M}: E_{\lambda}\left(M^{\wedge_{b} \curvearrowright}\right) \rightarrow M^{\wedge_{b}}$ such that $\Upsilon_{M}\left(\widetilde{\theta}_{\lambda}^{-1}(h) \cdot m\right)=\theta_{b}^{-1}(h) \cdot \Upsilon_{M}(m)$ for all $h \in \mathrm{C}(\underline{H})$.

Recall the notation used in Subsection 2.3 and, in particular, isomorphisms

$$
\Theta^{i}:\left.\mathcal{H}_{h}^{\wedge \mathcal{L}}\right|_{\mathcal{L}} \rightarrow \mathrm{C}\left(\left.\underline{\mathcal{H}}_{h}^{\wedge}\right|_{\underline{\mathcal{L}}}\right)^{\Xi}\left(U_{i}\right), i=1,2,
$$


and an element

$$
X_{12} \in \mathfrak{z}^{h}\left(\mathrm{C}\left(\underline{\mathcal{H}}_{h}^{\wedge} \underline{\mathcal{L}}_{\underline{\mathcal{L}}}\right)^{\Xi}\right)\left(U_{12}\right),
$$

with $\Theta^{1}=\exp \left(\operatorname{ad} X_{12}\right) \Theta^{2}$.

Our goal will be to produce $\Upsilon_{M}$ from $\exp \left(\left(\Theta^{2}\right)^{-1}\left(X_{12}\right)\right)$. A rough idea here is to make $\exp \left(\left(\Theta^{2}\right)^{-1}\left(X_{12}\right)\right)$ act on $E_{\lambda}\left(M^{\wedge_{b} \odot}\right)$ by "setting $h=1$ ". However, it is unclear why the infinite sum $\exp \left(\left(\Theta^{2}\right)^{-1}\left(X_{12}\right)\right) m$ has to converge for any $m \in E_{\lambda}\left(M^{\wedge_{b} \odot}\right)$. In fact, we can make the sum converge but we need to change our setting for this. Namely, we will replace $\mathbb{C}$ with the field $\left.R:=\mathbb{C}\left[t^{-1}, t\right]\right]$ of formal Laurent series and a point $(b, \lambda)$ with $(b, \lambda / t)$. In the next subsection we will see that the required sum converges and define an embedding similar to (6). Then we will introduce functors $\operatorname{res}_{b, \lambda / t}, \operatorname{Res}_{b, \lambda / t}$ and establish an embedding $\operatorname{res}_{b, \lambda / t} \hookrightarrow \operatorname{Res}_{b, \lambda / t} \cong \operatorname{Res}_{b, 0 / t}$. Next, in Subsection 3.3 we will see that the embedding $\operatorname{res}_{b, \lambda / t} \hookrightarrow \operatorname{Res}_{b, 0 / t}$ gives rise to an embedding $\operatorname{res}_{b, \lambda} \hookrightarrow \operatorname{Res}_{b, 0}$.

3.2. Functors $\operatorname{Res}_{b, \lambda / t}, \operatorname{res}_{b, \lambda / t}$. Set $\left.R:=\mathbb{C}\left[t^{-1}, t\right]\right]$. Consider the $R$-algebra $R\left[\mathfrak{h}^{*} / W\right]:=R \otimes_{\mathbb{C}} \mathbb{C}\left[\mathfrak{h}^{*} / W\right]$. It has a maximal ideal $\mathfrak{m}_{\lambda / t}$ corresponding to $\lambda / t$, so we can form the completion $R\left[\mathfrak{h}^{*} / W\right]^{\wedge_{\lambda / t}}$ with respect to this ideal. Consider the algebras $H_{R}:=R \otimes H, \underline{H}_{R}$ and the sheaves $\left.\mathcal{H}_{R, h}^{\wedge \mathcal{L}}\right|_{\mathcal{L}}$, etc. The isomorphisms $\Theta^{1}, \Theta^{2}$ naturally extend to isomorphisms of the algebras of sections of the corresponding sheaves. Now form the algebras $H_{R}^{\wedge_{\lambda / t}}, \underline{H}_{R}^{\wedge_{\lambda / t}}$ similarly to the above. The isomorphism $\Theta^{2}$ induces an isomorphism $\widetilde{\theta}_{\lambda / t}: H_{R}^{\wedge^{\lambda / t}} \rightarrow \mathrm{C}\left(\underline{H}_{R}^{\lambda / t}\right)$. Similarly, we have the completions $H_{R}^{\wedge_{b}}, \underline{H}_{R}^{\wedge_{b}}$ and their isomorphism $\theta_{b}$ induced by $\Theta^{1}$.

The algebras considered above come with the " $t$-Euler" derivation $t \frac{d}{d t}$. Since $\Theta^{1}, \Theta^{2}$ are defined over $\mathbb{C}$, we see that they intertwine $t \frac{d}{d t}$. It follows that $\theta_{b}, \widetilde{\theta}_{\lambda / t}$ also intertwine these derivations.

Now let $M \in \mathcal{O}$. Consider the $\left.H_{R^{-m o d u l e}} M\left[t^{-1}, t\right]\right]$ and its completion $\left.M\left[t^{-1}, t\right]\right]^{\wedge_{b}}$ in the $\mathfrak{m}_{b}$-adic topology, where we view $\mathfrak{m}_{b}$ as a maximal ideal in $R[\mathfrak{h} / W]$. We equip $\left.M\left[t^{-1}, t\right]\right]^{\wedge_{b}}$ with a topology taking $U_{k, l}:=t^{k} M^{\wedge_{b}}[[t]]+$ $\left.\mathfrak{m}_{b}^{l} M\left[t^{-1}, t\right]\right], k \in \mathbb{Z}, l \in \mathbb{Z}_{\geqslant 0}$ for the fundamental system of neighborhoods of 0 . In other words, a sequence $m_{i}$ of elements in $\left.M\left[t^{-1}, t\right]\right]^{\wedge_{b}}$ converges if the images of $m_{i}$ in $\left.M\left[t^{-1}, t\right]^{\wedge_{b}} / \mathfrak{m}_{b}^{n}=M / \mathfrak{m}_{b}^{n}\left[t^{-1}, t\right]\right]$ converge in the $t$-adic topology for all $n$. We can define the $H_{R^{-}}$submodule $\left.E_{\lambda / t}\left(M\left[t^{-1}, t\right]\right]^{\wedge_{b}}\right)$ similarly to the above. Our goal now will be to produce a certain family of maps $\left.\left.E_{\lambda / t}\left(M\left[t^{-1}, t\right]\right]^{\wedge_{b}}\right) \rightarrow M\left[t^{-1}, t\right]\right]^{\wedge_{b}}$.

Define a derivation $d$ of $H_{R, h}$ by $d . w=0, d . x_{\alpha}=0, d . y_{\alpha}=y_{\alpha}, d . h=h, d . t=-t$. The algebra $H_{R, h}$ acts on $\left.M\left[t^{-1}, t\right]\right]^{\wedge_{b}}$ via the homomorphism $H_{R, h} \rightarrow H_{R}$ given by $x_{\alpha} \mapsto x_{\alpha}, y_{a} \mapsto y_{a}, h \mapsto 1, w \mapsto w$. Consider the ideal $\mathfrak{m}$ in $\mathfrak{z}^{h}\left(H_{R, h}\right)$ corresponding to the point $(b, \lambda / t)$. Let $\left(H_{R, h}\right)_{d-f i n}, \widetilde{\mathcal{A}}$ denote the subalgebras of $d$-finite elements in $H_{R, h}, H_{R, h}^{\wedge_{b, \lambda / t}}$, where the latter stands for the completion of $H_{R, h}$ with respect to $\mathfrak{m}$. Of course, $\left(H_{R, h}\right)_{d-f i n}=\mathbb{C}\left[t^{ \pm 1}\right] \otimes H$. We also remark that the eigenvalues of $d$ on $\widetilde{\mathcal{A}}$ are all integral.

Proposition 3.3. For any $\left.m \in E_{\lambda / t}\left(M\left[t^{-1}, t\right]\right]^{\wedge_{b}}\right)$ the map

$$
\left.\left.\left(H_{R, h}\right)_{d-f i n} \rightarrow M\left[t^{-1}, t\right]\right]^{\wedge_{b}}\right), h \mapsto h . m
$$

extends uniquely to a continuous map $\left.\widetilde{\mathcal{A}} \rightarrow M\left[t^{-1}, t\right]\right]^{\wedge_{b}}$.

Proof. We need to show that for all $a \in \mathbb{Z}, n_{1}, n_{2} \in \mathbb{Z}_{\geqslant 0}$ there is $n$ such that $\left(H_{R, h} \mathfrak{m}^{n}\right)_{a} \cdot m \subset U_{n_{1}, n_{2}}$, where $\left(H_{R, h} \mathfrak{m}^{n}\right)_{a}$ denotes the subspace of all elements 
$f \in H_{R, h} \mathfrak{m}^{n}$ with $d(f)=$ af (we remark that $\mathfrak{m}$ is $d$-stable). First, let us define some $d$-stable filtration on $H_{R, h}$ that is equivalent to $H_{R, h} \mathfrak{m}^{n}$. Choose elements $f_{1}, \ldots, f_{k}$ generating the ideal of $b$ in $\mathbb{C}[\mathfrak{h}]^{W}$ and elements $g_{1}, \ldots, g_{r} \in R\left[\mathfrak{h}^{*}\right]^{W}$ generating the ideal of $\lambda / t$. The latter ideal is $d$-stable, so we may assume that all $g_{i}$ are eigenvectors for $d$ with some integral eigenvalues $\alpha_{1}, \ldots, \alpha_{r}$. The $R$-algebra $\mathfrak{z}^{h}\left(H_{R, h}\right)$ is finite over its subalgebra generated by $f_{i}, g_{i}, i=1, \ldots, r$ and $h$. Let $F_{1}, \ldots, F_{l}$ be a finite set of generators that are eigenvectors for $d$ with eigenvalues, say, $\beta_{1}, \ldots, \beta_{l}$. Then it is easy to see that $H_{R, h} \mathfrak{m}^{n}$ is equivalent to the filtration $\mathfrak{m}_{i}$ defined as follows:

$$
\mathfrak{m}_{i}:=\sum_{j+k+s=i} f_{i_{1}} \ldots f_{i_{j}} \operatorname{Span}_{R}\left(F_{1}, \ldots, F_{l}\right) h^{s} g_{i_{1}^{\prime}} \ldots g_{i_{k}^{\prime}} .
$$

Consider a monomial $f:=h^{s} t^{q} f_{i_{1}} \ldots f_{i_{k}} F_{t} g_{i_{1}^{\prime}} \ldots g_{i_{l}^{\prime}} \in \mathfrak{m}_{i}$ such that $d$ acts on $f$ by $a$. The last condition can be rewritten as $s-q+\beta_{t}+\sum_{j=1}^{l} \alpha_{i_{j}^{\prime}}=a$. For sufficiently large $l$, say $l>l_{1}$, where $l_{1}$ depends only on $m$, we have $g_{i_{1}^{\prime}} \ldots g_{i_{1}^{\prime}} m=0$. So we may assume that $l \leqslant l_{1}$. Also, if $k \geqslant n_{2}$, then $\left.\left.f_{i_{1}} \ldots f_{i_{k}} M\left[t^{-1}, t\right]\right]^{\wedge_{b}} \subset \mathfrak{m}_{b}^{n_{2}} M\left[t^{-1}, t\right]\right]^{\wedge_{b}} \subset$ $U_{n_{1}, n_{2}}$. So we may assume that $k \leqslant n_{2}$. This means that $s \geqslant i-n_{2}-l_{1}$ and so $q \geqslant i-M$, where $M$ is some constant depending only on $n_{2}, l_{1}$. The $\mathbb{C}$-linear span of all vectors of the form $t^{-q} f . m$ for all monomials $f$ with $l \leqslant l_{1}, k \leqslant n_{2}$ is finite dimensional (recall that $h$ acts by 1 ) because the number of the possible monomials $f$ is finite. It follows that for sufficiently large $i$ we get $t^{q}\left(t^{-q} f . m\right) \in U_{n_{1}, n_{2}}$.

Set $A_{h}:=\left(\Theta^{2}\right)^{-1}\left(X_{12}\right)$. It is a $\left(\mathbb{C}^{\times}\right)^{2}$-equivariant element of $\left.\mathcal{H}_{h}^{\wedge \mathcal{L}}\right|_{\mathcal{L}}\left(U_{12}\right)$. Let us view $A_{h}$ as an element of $\widetilde{\mathcal{A}}$. By construction, $A_{h}$ is annihilated by $\frac{d}{d t}$ and hence lies in $\mathbb{C}$ modulo $\mathfrak{m}$. Subtracting the corresponding element of $\mathbb{C}$, we may assume that $A_{h} \in \mathfrak{m}$. Since $A_{h}$ is $\left(\mathbb{C}^{\times}\right)^{2}$-invariant as an element in $\left.\mathcal{H}_{h}^{\wedge \mathcal{L}}\right|_{\mathcal{L}}\left(U_{12}\right)$, it is annihilated by $d$. So the element $\exp \left(A_{h}\right) \in \widetilde{\mathcal{A}}$ is defined and is $d$-invariant as well. So it defines a linear map $\left.\left.E_{\lambda / t}\left(M\left[t^{-1}, t\right]\right]^{\wedge_{b}}\right) \rightarrow M\left[t^{-1}, t\right]\right]^{\wedge_{b}}$.

Moreover, let $f \in \mathrm{C}\left(\underline{H}_{R, h}\right)$. Then $f$ is $\underline{d}$-finite, where $\underline{d}$ is the derivation of $\mathrm{C}\left(\underline{H}_{R, h}\right)$ defined similarly to $d$. The isomorphisms $\Theta^{1}, \Theta^{2}: H_{R, h}^{\wedge_{b, \lambda / t}} \rightarrow \mathrm{C}\left(\underline{H}_{R, h}^{\wedge, \lambda / t}\right)$ both intertwine $d$ and $\underline{d}$. It follows that $\left(\Theta^{1}\right)^{-1}(f) \exp \left(A_{h}\right)=\exp \left(A_{h}\right)\left(\Theta^{2}\right)^{-1}(f)$ in $H_{R, h}^{\wedge_{b, \lambda} t}$ and so the actions of the two sides on $\left.E_{\lambda / t}\left(M\left[t^{-1}, t\right]\right]^{\wedge_{b}}\right)$ agree. But $\left(\Theta^{1}\right)^{-1}(f)$ acts as $\theta_{b}^{-1}\left(f_{1}\right)$, while $\left(\Theta^{2}\right)^{-1}(f)$ acts as $\widetilde{\theta}_{\lambda / t}^{-1}\left(f_{1}\right)$. Here $f_{1}$ is the image of $f$ in $\mathrm{C}\left(\underline{H}_{R}\right)$. We remark that any $t \frac{d}{d t}$-finite element of $\mathrm{C}\left(\underline{H}_{R}\right)$ has the form $f_{1}$. Set $\Upsilon_{M, t}(m):=\exp \left(A_{h}\right) . m$. We conclude that

$$
\theta_{b}^{-1}(h) \Upsilon_{M, t}(m)=\Upsilon_{M, t}\left(\widetilde{\theta}_{\lambda / t}^{-1}(h) m\right),
$$

for all $t \frac{d}{d t}$-finite (and then, automatically, for all) elements of $\mathrm{C}\left(\underline{H}_{R}\right)$.

So we get the map $\left.\left.\Upsilon_{M, t}: E_{\lambda / t}\left(M\left[t^{-1}, t\right]\right]^{\wedge_{b}}\right) \rightarrow M\left[t^{-1}, t\right]\right]^{\wedge_{b}}$. Thanks to (7), the image of this map is contained in $\left.\underline{E}_{\lambda / t}^{\theta}\left(M\left[t^{-1}, t\right]\right]^{\wedge_{b}}\right)$. We claim that $\Upsilon_{M, t}$ is a bijection $\left.\left.E_{\lambda / t}\left(M\left[t^{-1}, t\right]\right]^{\wedge_{b}}\right) \rightarrow \underline{E}_{\lambda / t}^{\theta}\left(M\left[t^{-1}, t\right]\right]^{\wedge_{b}}\right)$. Indeed, analogously to Proposition 3.3. one can prove that the action of $\left(\Theta^{1}\right)^{-1}\left(\mathrm{C}\left(\underline{H}_{R, h}\right)_{\underline{d}-f i n}\right)$ on $\left.\underline{E}_{\lambda / t}^{\theta}\left(M\left[t^{-1}, t\right]\right]^{\wedge_{b}}\right)$ extends to that of $\widetilde{\mathcal{A}}$. Then it is easy to see that the map $m \mapsto \exp \left(-A_{h}\right) \cdot m$ is inverse to $\Upsilon_{M, t}$.

Also, we remark that $\left.M\left[t^{-1}, t\right]\right]$ comes equipped with an endomorphism $t \frac{d}{d t}$. This endomorphism extends to $\left.M\left[t^{-1}, t\right]\right]^{\wedge}$ by continuity, the extension will be 
denoted by $\mathrm{eu}_{t}^{M}$. It is compatible with the derivation $t \frac{d}{d t}$ of $H_{R, h}$ in the sense that $\mathrm{eu}_{t}^{M}(f m)=\left(t \frac{d}{d t} f\right) m+f \mathrm{eu}_{t}^{M} m$ for all $\left.m \in M\left[t^{-1}, t\right]\right]^{\wedge_{b}}, f \in H_{R, h}$. It is easy to see that both $\left.E_{\lambda / t}\left(M\left[t^{-1}, t\right]\right]^{\wedge_{b}}\right)$ and $\left.\underline{E}_{\lambda / t}^{\theta}\left(M\left[t^{-1}, t\right]\right]^{\wedge_{b}}\right)$ are $\mathrm{eu}_{t}^{M}$-stable. Since $\frac{d}{d t} A_{h}=0$, we see that $\Upsilon_{M, t}$ intertwines the operators $\mathrm{eu}_{t}^{M}$.

Now let us define certain functors $\operatorname{Res}_{b, \lambda / t}, \operatorname{res}_{b, \lambda / t}: \mathcal{O} \rightarrow \underline{\mathcal{O}}_{R}^{+}$. Here $\underline{\mathcal{O}}_{R}^{+}$stands for the category of $\underline{H}_{R}^{+}$-modules $N$ equipped with an operator eu ${ }_{t}^{N}$ subject to the following conditions:

(1) $N$ is finitely generated over $R\left[\mathfrak{h}_{W}\right]$,

(2) the operators $y_{a}, a \in \mathfrak{h}_{W}$ act locally nilpotently on $N$,

(3) the operator eu $u_{t}^{N}$ is compatible with the derivation $t \frac{d}{d t}$ of $\underline{H}_{R}^{+}$.

Then we will establish the isomorphism $\operatorname{res}_{b, \lambda / t} \stackrel{\sim}{\rightarrow} \operatorname{Res}_{b, \lambda / t} \stackrel{\sim}{\rightarrow} \operatorname{Res}_{b, 0 / t}$.

Let us construct a functor $\operatorname{Res}_{b, \lambda / t}$.

Take a module $M \in \mathcal{O}$. Form the $\left.H_{R^{-m o d u l e}} M\left[t^{-1}, t\right]\right]^{\wedge_{b}}$ and the endomorphism $\mathrm{eu}_{t}^{M}$ of this module. Consider the $\mathrm{C}\left(\underline{H}_{R}^{\wedge_{b}}\right)$-module $\left.\left(\theta_{b}\right)_{*}\left(M\left[t^{-1}, t\right]\right]^{\wedge_{b}}\right)$. Set $\underline{\mathrm{eu}}_{t}^{M, \theta}:=$ $\left(\theta_{b}\right)_{*}\left(\mathrm{eu}_{t}^{M}\right)$. Since $\theta_{b}$ intertwines the derivations $t \frac{d}{d t}$, we see that $\underline{\mathrm{eu}}_{t}^{M, \theta}$ is compatible with $t \frac{d}{d t}$.

Consider the subspace

$$
\left.\zeta_{\lambda / t} \circ \underline{E}_{\lambda / t} \circ I^{-1} \circ\left(\theta_{b}\right)_{*}\left(M\left[t^{-1}, t\right]\right]^{\wedge_{b}}\right)
$$

in $\left.\left(\theta_{b}\right)_{*}\left(M\left[t^{-1}, t\right]\right]^{\wedge_{b}}\right)$. This subspace is $\underline{H}_{R}^{+}$-stable. Also this subspace is preserved by $\underline{\mathrm{eu}}_{t}^{M+}:=\underline{\mathrm{eu}}_{t}^{M, \theta}+\lambda / t$. Indeed, (8) consists of all elements that are annihilated by $y_{a}-\langle a, \lambda\rangle / t$ with $a \in \mathfrak{h} \underline{\underline{W}}$ and by some powers of $y_{a}$ with $a \in \mathfrak{h}_{\underline{W}}$. Both of these conditions are preserved by $\underline{\mathrm{eu}}_{t}^{M+}$.

Let us check that (8) lies in $\underline{\mathcal{O}}_{R}^{+}$. For this we will need the following lemma which is a ramification of Lemma 2.2 . Let $\underline{\mathcal{O}}_{R}^{\prime}$ denote the category of all $\underline{H}_{R}^{\prime}:=$ $\left.\mathbb{C}[\mathfrak{h}]\left[t^{-1}, t\right]\right]^{\wedge_{b}} \otimes_{\mathbb{C}[\mathfrak{h}]} \underline{H}$-modules $M^{\prime}$ that are finitely generated over $\left.\mathbb{C}[\mathfrak{h}]\left[t^{-1}, t\right]\right]^{\wedge_{b}}$ and come equipped with an operator $\mathrm{eu}_{t}^{M^{\prime}}$ that is compatible with $t \frac{d}{d t}$.

Lemma 3.4. The assignment $\left(M^{\prime}, \mathrm{eu}_{t}^{M^{\prime}}\right) \mapsto\left(\zeta_{\lambda / t} \circ \underline{E}_{\lambda / t}\left(M^{\prime}\right), \mathrm{eu}_{t}^{+}:=\mathrm{eu}_{t}^{M^{\prime}}+\lambda / t\right)$ defines an equivalence between $\underline{\mathcal{O}}_{R}^{\prime}$ and $\underline{\mathcal{O}}_{R}^{+}$. This equivalence does not depend on $\lambda$ up to an isomorphism.

Proof. An isomorphism $\zeta_{0} \circ \underline{E}_{0}\left(M^{\prime}\right) \rightarrow \zeta_{\lambda / t} \circ \underline{E}_{\lambda / t}\left(M^{\prime}\right)$ is given by $m \mapsto e^{\lambda / t} m$ (this map is well defined on $\zeta_{0} \circ \underline{E}_{0}\left(M^{\prime}\right)$-i.e., an a priori infinite sum is actually finite - because $\lambda$ acts locally nilpotently). So it remains to prove that $\zeta_{0} \circ \underline{E}_{0}\left(M^{\prime}\right)$ is finitely generated over $R\left[\mathfrak{h}_{\underline{W}}\right]$. The module $\zeta_{0} \circ \underline{E}_{0}\left(M^{\prime}\right)$ is a direct limit of modules from $\underline{\mathcal{O}}_{R}^{+}$and so $\underline{\mathrm{eu}}^{+}$acts locally finitely on $\zeta_{0} \circ \underline{E}_{0}\left(M^{\prime}\right)$. Also we remark that $\zeta_{0} \circ \underline{E}_{0}\left(M^{\prime}\right) \subset \zeta_{0}\left(M^{\prime}\right)$. The latter is a finitely generated $R\left[\left[\mathfrak{h}_{W}\right]\right]$-module because $M^{\prime}=R\left[\mathfrak{h}^{W}\right]^{\wedge_{b}} \otimes \zeta_{0}\left(M^{\prime}\right)$ is a finitely generated $R[\mathfrak{h}]^{\wedge_{b}}$-module. So the submodule of $\underline{\mathrm{eu}}^{+}$-finite vectors in $\zeta_{0}\left(M^{\prime}\right)$ is finitely generated over $R\left[\mathfrak{h}_{\underline{W}}\right]$. Since $\zeta_{0} \circ \underline{E}_{0}\left(M^{\prime}\right)$ is a submodule in that module, our claim follows.

So (8) is indeed an object of $\underline{\mathcal{O}}_{R}^{+}$. By $\operatorname{Res}_{b, \lambda / t}$ we denote a functor that assigns (8) to $M$. By Lemma 3.4 this functor does not depend on $\lambda$.

Now let us proceed to defining the functor $\operatorname{res}_{b, \lambda / t}$. Again, we consider the $H_{R^{-}}$module $\left.M\left[t^{-1}, t\right]\right]$ and then its completion $\left.M\left[t^{-1}, t\right]\right]^{\wedge_{b}}$. Consider the $H_{R^{-}}$ submodule $\left.E_{\lambda / t}\left(M\left[t^{-1}, t\right]\right]^{\wedge_{b}}\right)$ of $\left.M\left[t^{-1}, t\right]\right]^{\wedge_{b}}$. It is straightforward to see that this 
module is stable under $\mathrm{eu}_{t}^{M}$. Recall that $\tilde{\theta}_{\lambda / t}$ intertwines the derivations $t \frac{d}{d t}$. We have the operator $\underline{\mathrm{eu}}_{t}^{M}:=\left(\tilde{\theta}_{\lambda / t}\right)_{*}\left(\mathrm{eu}_{t}^{M}\right)$ on $\left.\left(\widetilde{\theta}_{\lambda / t}\right)_{*}\left(E_{\lambda / t}\left(M\left[t^{-1}, t\right]\right]^{\wedge_{b}}\right)\right)$ compatible with $t \frac{d}{d t}$.

Consider the subspace

$$
\left.\zeta_{\lambda / t} \circ I^{-1} \circ\left(\tilde{\theta}_{\lambda / t}\right)_{*}\left(E_{\lambda / t}\left(M\left[t^{-1}, t\right]\right]^{\wedge_{b}}\right)\right)
$$

in $\left.\left(\widetilde{\theta}_{\lambda / t}\right)_{*}\left(E_{\lambda / t}\left(M\left[t^{-1}, t\right]\right]^{\wedge b}\right)\right)$. It comes equipped with the operator $\underline{\mathrm{eu}}_{t}^{M+}$ defined similarly to $\underline{\mathrm{eu}}_{t}^{M+}$. Then we can define $\operatorname{res}_{b, \lambda / t}$ similarly to $\operatorname{Res}_{b, \lambda / t}$.

Recall that we have the isomorphism

$$
\left.\left.\Upsilon_{M, t}:\left(\widetilde{\theta}_{\lambda / t}\right)_{*}\left(E_{\lambda / t}\left(M\left[t^{-1}, t\right]\right]^{\wedge_{b}}\right)\right) \rightarrow \underline{E}_{\lambda / t} \circ\left(\theta_{b}\right)_{*}\left(M\left[t^{-1}, t\right]\right]^{\wedge_{b}}\right) .
$$

By the construction this isomorphism intertwines the operators $\underline{\mathrm{eu}}_{t}^{M+}, \underline{\mathrm{eu}}_{t}^{M+}$. Therefore it induces an isomorphism $\operatorname{res}_{b, \lambda / t} \stackrel{\sim}{\rightarrow} \operatorname{Res}_{b, \lambda / t}$.

Our conclusion is that $\operatorname{res}_{b, \lambda / t} \stackrel{\sim}{\rightarrow} \operatorname{Res}_{b, 0 / t}$.

3.3. An embedding $\operatorname{res}_{b, \lambda} \hookrightarrow \operatorname{Res}_{b, 0}$. First, let us relate $\operatorname{Res}_{b, 0 / t}$ and $\operatorname{Res}_{b, 0}$. We remark that $M^{\wedge_{b}}$ is nothing more than the 0-eigenspace for $\mathrm{eu}_{t}^{M}$ in $\left.M\left[t^{-1}, t\right]\right]^{\wedge_{b}}$. From here, tracking the constructions of $\operatorname{Res}_{b, 0 / t}, \operatorname{Res}_{b, 0}$, we see that $\operatorname{Res}_{b, 0}(M)$ is the 0-eigenspace of $\underline{\mathrm{eu}}_{t}^{M+}$ in (8). The latter subspace is $\underline{H}^{+}$-stable. Moreover, since the annihilator of $\mathfrak{h}^{m}$ in $\left(\theta_{b}\right)_{*} M^{\wedge_{b}}$ is finite dimensional for all $m$, we see that $\left.\underline{E}_{0} \circ\left(\theta_{b}\right)_{*}\left(M\left[t^{-1}, t\right]\right]^{\wedge_{b}}\right)=R \otimes \underline{E}_{0} \circ\left(\theta_{b}\right)_{*}\left(M^{\wedge_{b}}\right)$. Therefore, $\operatorname{Res}_{b, 0 / t}(M)=$ $R \otimes \operatorname{Res}_{b, 0}(M)$. In particular, $\operatorname{Res}_{b, 0}(M)=\operatorname{Res}_{b, 0 / t}(M)_{f i n} /(t-1)$, where the subscript " $f$ in" denotes the subspace of all $\mathrm{eu}_{t}^{M+}$-finite elements.

So we need to produce a functorial embedding $\operatorname{res}_{b, \lambda}(M) \hookrightarrow \operatorname{res}_{b, \lambda / t}(M)_{f i n} /$ $(t-1)$. For this we will need two technical lemmas concerning Euler elements.

Lemma 3.5. $\Theta^{2}(\mathrm{eu})-\underline{\mathrm{eu}} \in \mathbb{C} h$.

Proof. The center of $\underline{H}_{h}^{\wedge_{0}}$ coincides with $\mathbb{C}[[h]]$, this follows easily from the claim (see $[\mathrm{BG}]$ ) that the center of $\underline{H}$ is $\mathbb{C}$. Therefore the centers of $\underline{H}_{h}^{\wedge_{b}}$ and $\mathrm{C}\left(\underline{H}_{h}^{\wedge_{b}}\right)$ coincide with $\mathbb{C}[[h]]$. Let us show that

$$
\left[\Theta^{2}(\mathrm{eu}), \cdot\right]=[\underline{\mathrm{eu}}, \cdot] .
$$

The derivation $[\mathrm{eu}, \cdot]$ is the image of $1 \in \mathbb{C}$ under the $\mathbb{C}^{\times}$-action on $H_{h}$ given by $z . x=z x, z \cdot y=z^{-1} y, z \cdot w=w, z . h=h$. A similar claim holds for eu. (10) follows from the claim that $\Theta^{2}$ intertwines the corresponding $\mathbb{C}^{\times}$-actions. Now consider the $\mathbb{C}^{\times}$-actions induced by the gradings on $H, \underline{H}$. They are also intertwined by $\Theta^{2}$. Since both eu and eu have degree 2 with respect to these actions, we see that $\Theta^{2}(\mathrm{eu})-\underline{\mathrm{eu}} \in \mathbb{C} h$.

Lemma 3.6. Let $M$ be a $\underline{H}$-module. Then $\underline{\mathrm{eu}}$ acts as $\underline{\mathrm{eu}}^{+}+\lambda+\operatorname{dim} \mathfrak{h} \underline{W} / 2$ on $\zeta_{\lambda}(M)$.

Proof. Pick a basis $y_{1}, \ldots, y_{n}$ in such a way that $y_{1}, \ldots, y_{k}$ is a basis in $\mathfrak{h}_{\underline{W}}$, while $y_{k+1}, \ldots, y_{n}$ is a basis in $\mathfrak{h} \underline{\underline{W}}$. So we see that $\underline{\mathrm{eu}}=\underline{\mathrm{eu}}^{+}+\sum_{i=k+1}^{n} x_{i} y_{i}+\frac{n-k}{2}$. The element $\sum_{i=k+1}^{n} x_{i} y_{i}$ acts by $\sum_{i=k+1}^{n} x_{i}\left\langle\lambda, y_{i}\right\rangle=\lambda$ on $\zeta_{\lambda}(M)$. Hence our claim.

Pick a section $\varphi: \mathbb{C} / \mathbb{Z} \hookrightarrow \mathbb{C}$ of the natural projection $\mathbb{C} \rightarrow \mathbb{C} / \mathbb{Z}$. Define an embedding $\left.\iota: M^{\wedge_{b} \odot} \hookrightarrow M\left[t^{-1}, t\right]\right]^{\wedge_{b}}$ by sending a sum $\sum_{i \in \mathbb{Z}_{\geqslant 0}} m_{a, i}$ (in the notation of Subsection [3.1) to $\sum_{i \in \mathbb{Z} \geqslant 0} t^{\varphi(a)-a-i} m_{a, i}$. It is easy to see that this embedding 
induces an embedding $\iota: \operatorname{res}_{b, \lambda}(M) \hookrightarrow \operatorname{res}_{b, \lambda / t}(M)$. Moreover, the last embedding becomes an $\underline{H}^{+}$-module homomorphism if we modify the action of $\underline{H}^{+}$on the image by $w . \iota(m)=w \iota(m), x . \iota(m)=t^{-1} x \iota(m), y . \iota(m)=t y \iota(m), w \in \underline{W}, x \in \mathfrak{h}_{W}^{*}, y \in$ $\mathfrak{h}_{W}$. We remark that $\underline{\mathrm{eu}}^{+}+\underline{\mathrm{eu}}_{t}^{M+}$ acts locally finitely on $\iota\left(\operatorname{res}_{b, \lambda}(M)\right)$. Indeed, thanks to Lemmas 3.5, 3.6, $\underline{\mathrm{eu}}^{+}+\underline{\mathrm{eu}}_{t}^{M+}$ coincides (up to adding a scalar) with the operator on $\operatorname{res}_{b, \lambda}(M)$ induced by eu $+\mathrm{eu}_{t}^{M}$. But if $m=\sum_{i \geqslant 0} m_{a, i}$, then $\iota(m)$ is a generalized eigenvector for $\mathrm{eu}+\mathrm{eu}_{t}^{M}$ with eigenvalue $\varphi(a)$. In particular, since $\mathrm{eu}^{+}$ acts locally finitely on any object in $\underline{\mathcal{O}}_{R}^{+}$, we see that $\iota\left(\operatorname{res}_{b, \lambda}(M)\right) \subset \operatorname{res}_{b, \lambda / t}(M)_{\text {fin }}$. Now consider the induced map $\mathbb{C}\left[t^{-1}, t\right] \otimes \iota\left(\operatorname{res}_{b, \lambda}(M)\right) \rightarrow \operatorname{res}_{b, \lambda / t}(M)_{\text {fin }}$. The kernel of this map is stable with respect to $\underline{\mathrm{eu}}^{+}+\underline{\mathrm{eu}}_{t}^{M+}$ and the action of this operator on $\mathbb{C}\left[t^{-1}, t\right] \otimes \iota\left(\operatorname{res}_{b, \lambda}(M)\right)$ is locally finite. So let $v$ be an eigenvector in the kernel. But the kernel is stable under the multiplication by elements from $\mathbb{C}\left[t^{-1}, t\right]$ as well. However, it is easy to see that for an appropriate $k$ we have $t^{k} v \in$ $\iota\left(\operatorname{res}_{b, \lambda}(M)\right)$, contradiction. So the map $\mathbb{C}\left[t^{-1}, t\right] \otimes \iota\left(\operatorname{res}_{b, \lambda}(M)\right) \rightarrow \operatorname{res}_{b, \lambda / t}(M)_{f i n}$ is injective. Taking the quotient by $t-1$, we get a required embedding $\operatorname{res}_{b, \lambda}(M) \hookrightarrow$ $\operatorname{res}_{b, \lambda / t}(M)_{f i n} /(t-1)$.

3.4. An embedding $\operatorname{res}_{0, \lambda} \hookrightarrow \operatorname{res}_{b, \lambda}$. Tracking the construction of $\operatorname{res}_{b, \lambda}$ we see that we need to prove the following claim:

(*) There is a functorial embedding $E_{\lambda}\left(M^{\wedge_{0}}\right) \hookrightarrow E_{\lambda}\left(M^{\wedge_{b} \odot}\right)$.

In fact we will show a weaker result.

Proposition 3.7. There is a sufficiently small $W$-stable neighborhood $U$ of zero in $\mathfrak{h}$ such that for all $b \in U$ there is a functorial homomorphism $E_{\lambda}\left(M^{\wedge_{0}}\right) \rightarrow$ $E_{\lambda}\left(M^{\wedge}{ }_{b} \odot\right)$ that is injective when $M$ is projective.

Proof. Let $U$ be a convex $W$-stable neighborhood of 0 in $\mathfrak{h}$. Set $H(U):=\mathbb{C}[U] \otimes_{\mathbb{C}[\mathfrak{h}]}$ $H$, where $\mathbb{C}[U]$ stands for the algebra of analytic functions on $U$. For $M \in \mathcal{O}$ set $M(U):=\mathbb{C}[U] \otimes_{\mathbb{C}[\mathfrak{h}]} M$. We can define $E_{\lambda}(M(U))$ similarly to the above. We have a natural homomorphism $E_{\lambda}(M(U)) \hookrightarrow E_{\lambda}\left(M^{\wedge_{0}}\right)$ (restricting a section to the formal neighborhood of 0 ).

Lemma 3.8. The natural map $M(U) \rightarrow M^{\wedge_{0}}$ is an embedding for sufficiently small $U$.

Proof. Since both $M \mapsto M^{\wedge_{0}}, M \mapsto M(U)$ are exact functors, it is enough to check the claim when $M$ is irreducible. Since there are only finitely many irreducibles, it is enough to check the claim for any fixed $M$. Here it follows easily from the observation that $M(U)$ is a finitely generated and hence Noetherian $\mathbb{C}[U]$-module.

Also, we have a homomorphism $E_{\lambda}(M(U)) \rightarrow E_{\lambda}\left(M^{\wedge_{b}}\right)$ (the restriction to a formal neighborhood of $b$ ) for any $b \in U$. Any section $s$ of $M(U)$ is a sum $\sum_{a \in \mathbb{C}} \sum_{i=0}^{\infty} m_{a, i}$ (where the meaning of $m_{a, i}$ is the same as in the definition of - $\bigcirc$ in Subsection (3.1) that converges on $U$. It follows that the image of the restriction embedding $M(U) \hookrightarrow M^{\wedge_{b}}$ lies in $M^{\wedge_{b} \odot}$. So $E_{\lambda}(M(U))$ actually maps to $E_{\lambda}\left(M^{\wedge}{ }_{b} \odot\right)$.

Now we claim that for sufficiently small $U$ the embedding $E_{\lambda}(M(U)) \rightarrow E_{\lambda}\left(M^{\wedge_{0}}\right)$ is actually an isomorphism. The category $\mathcal{O}$ is a length category, has enough projectives, and the number of projectives is finite. The functor $M \mapsto E_{\lambda}(M(U))$ is 
obviously left exact, while the functor $M \mapsto E_{\lambda}\left(M^{\wedge_{0}}\right)$ is exact. Recall that the latter follows from the fact that res $_{0, \lambda}$ is exact; see [BE], Subsection 3.5. Therefore the embedding $E_{\lambda}(M(U)) \hookrightarrow E_{\lambda}\left(M^{\wedge_{0}}\right)$ is an isomorphism if and only if it is an isomorphism for any projective $M$.

Now it is known (see GGOR) that any projective has a filtration by standard modules and, in particular, is a free $\mathbb{C}[\mathfrak{h}]$-module. The action of $y_{a}$ gives rise to a flat $W$-equivariant connection on this bundle. The connection has regular singularities on the reflection hyperplanes. Suppose that $v$ is an element of $M^{\wedge_{0}}$ such that $\left(y_{a}-\langle\lambda, a\rangle\right) v=u^{\prime}(a)$ for some linear map $u^{\prime}: \mathfrak{h} \rightarrow M(U)$. Since the connection given by $a \mapsto y_{a}$ has regular singularities, we see that $v$ extends to some smaller neighborhood $U^{\prime}$ of 0 . But the module $E_{\lambda}\left(M^{\wedge_{0}}\right)$ is finitely generated. So using an easy induction and shrinking $U$ if necessary, we see that all generators of $E_{\lambda}\left(M^{\wedge_{0}}\right)$ extend to $U$ proving our claim.

Since any projective module is free over $\mathbb{C}[\mathfrak{h}]$, we see that the natural map $M(U) \rightarrow M^{\wedge_{b}}$ is injective, provided $M$ is projective.

3.5. Completion of the proof. Let us complete the proof of the theorem. We have a homomorphism

$$
\operatorname{res}_{\lambda} \cong \operatorname{res}_{0, \lambda} \rightarrow \operatorname{res}_{b, \lambda} \hookrightarrow \operatorname{Res}_{b, \lambda} \cong \operatorname{Res}_{b}
$$

for $b$ sufficiently close to 0 . But all functors $\operatorname{Res}_{b}$ are isomorphic (see [BE], Subsection 3.7) so we have a homomorphism $\operatorname{res}_{\lambda} \rightarrow \operatorname{Res}_{b}$ for all $b$. Moreover, $\operatorname{res}_{\lambda}(M) \hookrightarrow$ $\operatorname{Res}_{b}(M)$ for any projective $M$. As Bezrukavnikov and Etingof checked in BE], Subsection 3.6, on the level of the Grothendieck groups the functors $\operatorname{res}_{\lambda}$, $\operatorname{Res}_{b}$ are the same $\left(K_{0}(\mathcal{O})=K_{0}(W-\operatorname{Mod}), K_{0}\left(\underline{\mathcal{O}}^{+}\right)=K_{0}(\underline{W}-\operatorname{Mod})\right.$ and both $\operatorname{Res}_{b}$, res $_{\lambda}$ produce the restriction map induced by the embedding $\underline{W} W$ ). $\operatorname{Sores}_{\lambda}(M), \operatorname{Res}_{b}(M)$ have the same class in the Grothendieck group for any $M$. In particular, $\operatorname{res}_{\lambda}(M)=$ $\operatorname{Res}_{b}(M)$ for projective $M$. Now we have a natural transformation of two exact functors that is an isomorphism on projectives. Such a transformation is necessarily an isomorphism.

\section{ISOMORPHISM OF THE INDUCTION FUNCTORS}

First, we define auxiliary functors

$$
\begin{array}{r}
\operatorname{Ind}_{b, 0}(N)=E_{0} \circ\left(\theta_{b}\right)_{*}^{-1} \circ I \circ\left(\zeta_{0} \circ \underline{E}_{0}\right)^{-1}(N), \\
\operatorname{ind}_{b, \lambda}(N)=E_{0}\left(\left(\left(\widetilde{\theta}_{\lambda}\right)_{*}^{-1} \circ I \circ \zeta_{\lambda}^{-1}(N)\right)^{\wedge_{b}}\right)
\end{array}
$$

from $\underline{\mathcal{O}}^{+} \rightarrow \widetilde{\mathcal{O}}$, where we consider $\zeta_{0} \circ \underline{E}_{0}$ as an equivalence $\underline{\mathcal{O}}^{\wedge_{b}} \rightarrow \underline{\mathcal{O}}^{+}$. We remark that we do not need to apply $\bullet$ in the definition of ind ${ }_{b, \lambda}$. Indeed, $E_{0}\left(M^{\prime}\right) \subset M^{\prime \odot}$ for any topological $H$-module because eu acts locally finitely on any object of $\widetilde{\mathcal{O}}$.

As in Subsection 3.1 one shows that $\operatorname{Ind}_{b, 0} \cong \operatorname{Ind}_{b}$, while $\operatorname{ind}_{0, \lambda} \cong \operatorname{ind}_{\lambda}$. Then, similarly, to Subsection 3.5, it is enough to show that there are

(A) a homomorphism $\operatorname{ind}_{0, \lambda} \rightarrow \operatorname{ind}_{b, \lambda}$ that is an embedding on projectives (for $b$ sufficiently close to 0$)$

(B) and an embedding $\operatorname{ind}_{b, \lambda} \hookrightarrow \operatorname{Ind}_{b, 0}$.

Lemma 4.1. There is a natural transformation $\operatorname{ind}_{0, \lambda} \rightarrow \operatorname{ind}_{b, \lambda}$ as in $(\mathrm{A})$. 
Proof. The proof closely follows that of Proposition 3.7. We need to show that for all $b$ sufficiently close to 0 there is a functorial homomorphism $E_{0}\left(M^{\wedge_{0}}\right) \rightarrow E_{0}\left(M^{\wedge_{b}}\right)$ for any $M \in \mathcal{O}^{\lambda}$, and that this homomorphism is an embedding whenever $M$ is projective. This is done exactly as in the proof of Proposition 3.7, the only two claims that we need to check are that $\mathcal{O}^{\lambda}$ is a length category with enough projectives, and that any projective is a free $\mathbb{C}[\mathfrak{h}]$-module. For a $\underline{W}$-module $\mu$ one can define the standard object $\Delta^{\lambda}(\mu)=H \otimes_{S \mathfrak{h} \# \underline{W}} \mu \cong \mathbb{C}[\mathfrak{h}] \otimes\left(\mathbb{C} W \otimes_{\mathbb{C} W} \mu\right)$, where $S \mathfrak{h}$ acts on $\mu$ via $\lambda$. The functor $\left(\widetilde{\vartheta}_{\lambda}\right)_{*}^{-1} \circ I \circ \zeta_{\lambda}^{-1} \cong\left(\widetilde{\theta}_{\lambda}\right)_{*}^{-1} \circ I \circ \zeta_{\lambda}^{-1}$ defines an equivalence $\underline{\mathcal{O}}^{+} \rightarrow \mathcal{O}^{\lambda}$. It is easy to check that this equivalence maps standards to standards. Since any projective in $\underline{\mathcal{O}}^{+}$admits a filtration, whose quotients are standards, we see that any projective in $\mathcal{O}^{\lambda}$ is free as a $\mathbb{C}[\mathfrak{h}]$-module.

To establish an embedding $\operatorname{ind}_{b, \lambda} \hookrightarrow \operatorname{Ind}_{b, 0}$ we argue as in Subsections 3.2 and 3.3. Namely, we introduce a category $\widetilde{\mathcal{O}}_{R}$ of certain $H_{R}$-modules equipped with Euler operators, compare with the definition of $\underline{\mathcal{O}}_{R}^{+}$in Subsection 3.2. Then we construct functors $\operatorname{Ind}_{b, \lambda / t}, \operatorname{ind}_{b, \lambda / t}: \underline{\mathcal{O}}^{+} \rightarrow \widetilde{\mathcal{O}}_{R}$ as follows:

$$
\begin{aligned}
& \operatorname{Ind}_{b, \lambda / t}(N)=E_{0} \circ\left(\theta_{b}\right)_{*}^{-1} \circ I \circ\left(\zeta_{\lambda / t} \circ \underline{E}_{\lambda / t}\right)^{-1}(R \otimes N), \\
& \operatorname{ind}_{b, \lambda / t}(N)=E_{0}\left(\left(\left(\widetilde{\theta}_{\lambda / t}\right)_{*}^{-1} \circ I \circ \zeta_{\lambda / t}(R \otimes N)\right)^{\wedge_{b}}\right) .
\end{aligned}
$$

To get an operator $\mathrm{eu}_{t}^{N}$ on, say, $\operatorname{Ind}_{b, \lambda / t}$, we reverse the procedure of obtaining $\underline{\mathrm{eu}}_{t}^{M+}$ from $\mathrm{eu}_{M}^{t}$; see Subsection 3.2. We also remark that in (13) we view $\zeta_{\lambda / t} \circ \underline{E}_{\lambda / t}$ as an equivalence $\underline{\mathcal{O}}_{R}^{\prime} \stackrel{\sim}{\rightarrow} \underline{\mathcal{O}}_{R}^{+}$; see Subsection 3.2

By Lemma 3.4. Ind $\operatorname{In}_{b, 0 / t} \cong \operatorname{Ind}_{b, \lambda / t}$. Next, similarly to the corresponding argument in Subsection 3.2, we can construct a map

$$
\operatorname{ind}_{b, \lambda / t}(N) \rightarrow\left(\theta_{b}\right)_{*}^{-1} \circ I \circ\left(\zeta_{\lambda / t} \circ \underline{E}_{\lambda / t}\right)^{-1}(R \otimes N) .
$$

This map is obtained by applying $\exp \left(X_{12}\right)$. As in Subsection 3.2, this map gives rise to an isomorphism $\operatorname{ind}_{b, \lambda / t} \stackrel{\sim}{\longrightarrow} \operatorname{Ind}_{b, \lambda / t}$.

A relation between the functors $\operatorname{Ind}_{b, 0}$ and $\operatorname{Ind}_{b, 0 / t}$ is completely analogous to that between $\operatorname{Res}_{b, 0}$ and $\operatorname{Res}_{b, 0 / t}$ (see Subsection 3.3). Namely, $\operatorname{Ind}_{b, 0 / t}(N)=R \otimes$ $\operatorname{Ind}_{b, 0}(N)$, and $\operatorname{Ind}_{b, 0}(N)$ is the quotient of the submodule of eu $+t \frac{d}{d t}$-finite elements in $\operatorname{Ind}_{b, 0 / t}(N)$ by $t-1$.

Now let us relate $\operatorname{ind}_{b, \lambda}$ to $\operatorname{ind}_{b, \lambda / t}$.

Set $M:=\left(\widetilde{\theta}_{\lambda}\right)_{*}^{-1} \circ I \circ \zeta_{\lambda}^{-1}(N)$. First of all, let us identify $M_{t}:=\left(\widetilde{\theta}_{\lambda}\right)_{*}^{-1} \circ I \circ$ $\zeta_{\lambda / t}^{-1}(R \otimes N)$ with $R \otimes M$. Namely, it is easy to see that $M_{t}$ gets identified with $R \otimes M$ if we modify the $H_{R}$-module structure on $R \otimes M$ as follows: $t \cdot m=t m, w \cdot m=$ $w m, x \cdot m=t x m, y \cdot m=t^{-1} y m, m \in M, w \in W, x \in \mathfrak{h}^{*}, y \in \mathfrak{h}$, where in the 1.h.s. we have the new action of $H_{R}$, and in the r.h.s. the action is standard. Under this identification $M_{t}^{\wedge_{b}}$ gets identified with $(R \otimes M)^{\wedge_{b t}}:=R[\mathfrak{h}]^{\wedge_{b t}} \otimes_{\mathbb{C}[\mathfrak{h}]} M$. So we need to relate the $H$-module $E_{0}\left(M^{\wedge_{b}}\right)$ to the $H_{R}$-module $E_{0}\left((R \otimes M)^{\wedge_{b t}}\right)$.

The module $(R \otimes M)^{\wedge_{b t}}$ comes equipped with an Euler operator $\mathrm{eu}_{t}^{M}$ induced from $t \frac{d}{d t}$ on $R \otimes M$. We remark that the maximal ideal $\mathfrak{m}_{b t} \subset R[\mathfrak{h}]^{W}$ is stable with respect to $[\mathrm{eu}, \cdot]+t \frac{d}{d t}$. Consider the quotient $M_{n}:=(R \otimes M) / \mathfrak{m}_{b t}^{n}$. We want to understand the structure of the operator $\mathrm{eu}+\mathrm{eu}_{t}^{M}$ on $M_{n}$. Recall the section $\varphi: \mathbb{C} / \mathbb{Z} \rightarrow \mathbb{C}$ chosen in Subsection 3.3 
Lemma 4.2. There are finitely many elements $\beta_{1}, \ldots, \beta_{k} \in \varphi(\mathbb{C} / \mathbb{Z})$ with $\beta_{i}-$ $\beta_{j} \notin \mathbb{Z}$ such that the $\mathrm{eu}+\mathrm{eu}_{t}^{M}$-finite part of $M_{n}$ is the direct sum of generalized eigenspaces of $\mathrm{eu}+\mathrm{eu}_{t}^{M}$ with eigenvalues $\beta_{i}+n, n \in \mathbb{Z}$. All generalized eigenspaces are finite dimensional. Moreover, if $M_{n}^{0}$ denotes the sum of generalized eigenspaces with eigenvalues $\beta_{1}, \ldots, \beta_{k}$, then the natural homomorphism $R \otimes M_{n}^{0} \rightarrow M_{n}$ is an isomorphism.

Proof. Consider the $\mathbb{C}[[t]] \otimes H$-module $\mathbb{C}[[t]] \otimes M$ that maps naturally to $R \otimes$ $M$. Then we have $R \otimes_{\mathbb{C}[[t]]} M_{n}^{+} \stackrel{\sim}{\rightarrow} M_{n}$, where $M_{n}^{+}:=(\mathbb{C}[[t]] \otimes M) / \mathfrak{m}_{b t}^{n}$. The operator eu $+\mathrm{eu}_{M}^{t}$ also acts naturally on $M_{n}^{+}, R \otimes_{\mathbb{C}[[t]]} M_{n}^{+}$and the identification $R \otimes_{\mathbb{C}[[t]]} M_{n}^{+} \stackrel{\sim}{\longrightarrow} M_{n}$ intertwines the corresponding operators. The $\mathbb{C}[[t]]$-module $M_{n}^{+}$is finitely generated because $\mathbb{C}[[t]] \otimes M$ is finitely generated over $\mathbb{C}[[t]] \otimes \mathbb{C}[\mathfrak{h}]$. Hence $M_{n}^{+}$is complete in the $t$-adic topology. All subspaces $t^{m} M_{n}^{+}$are eu $+\mathrm{eu}_{t}^{M_{-}}$ stable. The claim of the lemma follows easily from the observation that all quotients $M_{n}^{+} / t^{m} M_{n}^{+}$are finite dimensional over $\mathbb{C}$ and that the multiplication by $t$ increases the eigenvalue of $\mathrm{eu}+\mathrm{eu}_{t}^{M}$ by 1 .

The proof also shows that the subspace $\left(M_{n}^{+}\right)_{f i n}$ of eu $+\mathrm{eu}_{M^{t}}$-finite vectors in $M_{n}^{+}$coincides with $(\mathbb{C}[t] \otimes M) / \mathfrak{m}_{b t}^{n}$ embedded naturally into $M_{n}^{+}$(the natural map is an embedding because $M_{n}=\mathbb{C}[[t]] \otimes_{\mathbb{C}[t]}(\mathbb{C}[t] \otimes M) / \mathfrak{m}_{b t}^{n}$ and the torsion submodule of the $\mathbb{C}[t]$-module $(\mathbb{C}[t] \otimes M) / \mathfrak{m}_{b t}^{n}$ is supported at 0 thanks to the operator eu $\left.+\mathrm{eu}_{t}^{M}\right)$.

Let us identify $M_{n}^{0}$ with $M / \mathfrak{m}_{b}^{n}$. For $m \gg 0$ the space $t^{m} M_{n}^{0}$ lies in the torsionfree part of the $\mathbb{C}[[t]]$-module $M_{n}^{+}$. Then we just consider $t^{m} M_{n}^{0}$ as a subspace in $(\mathbb{C}[t] \otimes M) / \mathfrak{m}_{b t}^{n}$ and restrict the natural projection (=the quotient by $\left.t-1\right)$ $(\mathbb{C}[t] \otimes M) / \mathfrak{m}_{b t}^{n} \rightarrow M / \mathfrak{m}_{b}^{n}$ to $t^{m} M_{n}^{0}$. From Lemma 4.2 it follows easily that this map is a bijection. We remark that the bijection $M_{n}^{0} \rightarrow M / \mathfrak{m}_{b}^{n}$ is compatible with the natural projections $M_{n+1}^{0} \rightarrow M_{n}^{0}, M / \mathfrak{m}_{b}^{n+1} \rightarrow M / \mathfrak{m}_{b}^{n}$ (the claim that the first map is surjective is an easy corollary of Lemma 4.2).

It follows that $M^{\wedge_{b}}$ gets identified with the sum of generalized eigenspaces of elements of $\varphi(\mathbb{C} / \mathbb{Z})$ in $M_{t}^{\wedge b t}$. This is an $H$-module identification (where $H$ acts on the latter space by $\left.x \mapsto t^{-1} x, y \mapsto t y, w \mapsto w\right)$. From here it is easy to see that $E_{0}\left(M^{\wedge_{b}}\right)$ gets embedded into $E_{0}\left(M^{\wedge_{b t}}\right)$. This embedding produces the embedding ind $_{b, \lambda} \hookrightarrow \operatorname{Ind}_{b, 0}$ that we need.

\section{ACKNOWLEDGEMENTS}

The author is grateful to P. Etingof and I. Gordon for stimulating discussions. The author also wants to thank the referee for useful remarks on an earlier version of this paper.

\section{REFERENCES}

[BE] Roman Bezrukavnikov and Pavel Etingof, Parabolic induction and restriction functors for rational Cherednik algebras, Selecta Math. (N.S.) 14 (2009), no. 3-4, 397-425, DOI 10.1007/s00029-009-0507-z. MR2511190(2010e:20007)

[BG] Kenneth A. Brown and Iain Gordon, Poisson orders, symplectic reflection algebras and representation theory, J. Reine Angew. Math. 559 (2003), 193-216, DOI 10.1515/crll.2003.048. MR.1989650(2004i:16025)

[EG] Pavel Etingof and Victor Ginzburg, Symplectic reflection algebras, Calogero-Moser space, and deformed Harish-Chandra homomorphism, Invent. Math. 147 (2002), no. 2, 243-348, DOI 10.1007/s002220100171. MR1881922(2003b:16021) 
[GGOR] Victor Ginzburg, Nicolas Guay, Eric Opdam, and Raphaël Rouquier, On the category $\mathcal{O}$ for rational Cherednik algebras, Invent. Math. 154 (2003), no. 3, 617-651, DOI 10.1007/s00222-003-0313-8. MR2018786 (2005f:20010)

[L1] Ivan Losev, Completions of symplectic reflection algebras, Selecta Math. (N.S.) 18 (2012), no. 1, 179-251, DOI 10.1007/s00029-011-0071-1. MR2891864

[L2] I. Losev. Highest weight $\mathfrak{s l}_{2}$-categorifications I. Crystals. arXiv:1201.4493. Accepted by Math. Zeitschrift.

[S] Peng Shan, Crystals of Fock spaces and cyclotomic rational double affine Hecke algebras, Ann. Sci. Éc. Norm. Supér. (4) 44 (2011), no. 1, 147-182 (English, with English and French summaries). MR2760196 (2012c:20009)

Department of Mathematics, Northeastern University, 360 Huntington Avenue, Boston, Massachusetts 02115

E-mail address: i.loseu@neu.edu 